\title{
A CONTRIBUTION TO SONOGRAPH IMAGE QUALITY ESTIMATION USING POINT SPREAD FUNCTION
}

\author{
Ladislav Doležal, Jan Hálek \\ Palacky University Olomouc, Faculty of Medicine, Czech Republic: Institute of Medical Biophysics
}

\begin{abstract}
Summary: In medical sonography, sonograph image quality is an essential aspect for the safety of both patient and doctor. Its evaluation therefore requires an accurate and objective method for measurement. In this regard, a number of methods are in current use. Most of these are based on tissue mimicking phantom imaging. In contrast, we have used another principle based on Point Spread Function (PSF) analysis which is a product of the measuring system we have developed. In this case, the measured sonograph scans a small metallic ball target that moves in a water bath on a specified trajectory. The Region Of Interest (ROI) of the sonogram containing the ball target picture is digitised and the amplitude of the pixels analysed. The result is the PSF from which we calculate the lateral resolution (LR). For this purpose, we use our own original software. Using this method, we have to date been able to plot LR characteristics over the scanning plane. The method allows us to differentiate separate scanning lines and even multiple focal areas for dynamic focussing systems. It can detect malfunctions in dynamic focussing, size of aperture, time gain compensation function and/or transducer element failure. The procedure itself is not as easy or as fast to use as tissue mimicking phantoms or 3D signal to noise ratio evaluation, but it provides accurate and objective numeric parameters corresponding to the quality of image at any specified point over the whole scanning area. It is also a very powerful tool when used in combination with the other methods mentioned above.
\end{abstract}

Key words: Sonography; Quality; Resolution; Point spread function; Measurement

\section{Introduction}

Problems related to the safety of ultrasound application, judged from the point of view of patients, nursing and examining personnel, ultrasound biological effects have dominated the more than 50 years of ultrasound use in medicine; the direct effects of ultrasound energy on living tissue have been intensively focussed on for a considerable time. On the other hand, the dangers inherent in incorrect treatment resulting from erroneous diagnosis based on misinterpretation of the sonogram has only been taken into consideration in the last decade. Such misinterpretation has a number of sources. Firstly, artefacts. When evaluating the risks of image artefacts, it is necessary to differentiate objective and subjective factors.

a) Objective risk factors include:

- physical imaging artifacts,

- inadequate quality of imaging equipment caused by low technical standard, poor maintenance or age of the equipment.

b) Subjective factors relate to the skills of the examiner. These include:

- unfamiliarity with the physical mechanisms of ultrasound image creation, lack of skills in operating the equipment and hence inability to set the optimal working parameters,

- lack of knowledge of the topographic anatomy necessary for correct image interpretation,

- inborn characteristics of the observer, such as spatial imagination and the ability to abstract what is seen.

Physical artifacts here are based on the physical properties of ultrasound waves and the environment in which they are propagated. As such they are unequivocally definable according to laws of physics and to eliminate them, it is necessary to apply appropriate procedures and imaging methods. If these do not exist, the laws of physics must be accepted and taken into consideration. In this case eliminating the risks is totally dependent on the experience of the examiner and the above mentioned subjective characteristics.

On the other hand, the sonograph imaging quality is a factor completely dependent on the technical parameters of the equipment. In order to increase the imaging quality or eliminate imaging defects and thus reduce the risks, it is necessary to create a system for determining and objectively evaluating the relevant qualitative parameters (1).

This is very difficult and requires definition of the parameters of sonographic imaging quality, development of 
suitable measuring methods, procedures for their evaluation and creation of a graded system of sonograph quality criteria.

At present, commercial testing objects for B-mode (for example ATS 520, RMI 403 GS, CIRS etc.) are being produced. These contain defined non-homogeneities whose image is analyzed by subjective observation (5). To fulfill all important physical criteria for correct mimicking of the tissue (4), the construction has to be rather sophisticated although there are back up programs for their easier application and processing of the results - for example the Canadian UltraQ (2). This testing method is fast and relatively inexpensive, but is, however, burdened with a large error resulting from subjective assessment of image quality, even with the use of computer technology.

Another very interesting method (7), utilizes spatial analysis of the signal/noise ratio in a three-dimensional image of a special testing object to create the image characterization for signals with small amplitude. This method is suitable for fast orientation measurement and is substantially more objective than the ones mentioned earlier. Its only disadvantage is that it shows an integral parameter which is dependent on the depth and cannot therefore determine the lateral details of the image defect. Second, analysis of spatial image distortion and characterization of the system for high amplitude reflected signals is not possible. Both of these methods are primarily suitable for in situ screening studies which are not time consuming given the equipment workload. They do not, however, produce detailed objective information. Another simple and fast testing method is the control of homogeneity of the transducer field by common plane reflector, developed by the Sonora Co. (3) and called FirstCall 2000. This is suitable for fast detection of a defective spot on the electronic probe, but it is not, however, able to measure specific physical parameters such as, for example, resolution.

Another type of measurement that can be used for analyzing sonograph qualitative parameters is measurement of the characteristics of the radiated ultrasound field. This method, however, does not evaluate the image quality. It only determines the parameters of the transmitting ultrasound signal and is suitable mainly for controlling the radiated ultrasound intensity, or, possibly, its space and time distribution, which is significant for maintaining the allowed limits and for assessment of the effects of ultrasound energy in various types of tissue boundary. These are not the only measuring methods available. There are also mathematical models of the ultrasound field radiated by certain types of probes (6) and its temperature effects.

In short, quantitative and accurate evaluation of the image quality is very problemmatic and there exist few institutions world wide dealing with this issue using the methods mentioned above.

Our contribution is a new system of evaluating parameters for sonograph quality imaging in various imaging modes and methodical procedures for their quantitative, objective and accurate imaging. For this purpose, we evaluated the characteristics of the Point Spread Function (PSF) of the point reflector was carried out and the results were used for measuring spatial resolution using the newly developed measuring equipment.

\section{Methods}

We used a principle based on the PSF analysis, which is a product of the measuring system we have developed. The measured sonograph scans a small metallic ball target that moves in a water bath on a specified trajectory. The bath is filled with degassed water and the walls are fitted wih absorbant material. The positioning system has a ball target holder, designed according to instructions given in the IEC. The ball target consists of a small steel sphere, a laser welded to a tiny platinum wire which is fixed in a holder. The shape of the wire ensures that the sphere is oriented in front of the transducer in the scanned plane with the welding point in the distal position. The platinum wire is strong enough to eliminate any movement of the ball target during displacement in the water bath due to hydrodynamic forces. $3 \mathrm{D}$ positioning is arranged using three stepper motors connected to precise support screws. The motors are driven by a computer controlled power unit. The video signal from the test US scanner is driven to Frame Grabber NI PCI1411 (National Instruments), digitalized and Region Of Interest (ROI) is stored after on-line evaluation. The system selects the video frame containing the peak amplitude for each measurement point in the scanning plane to derive the PSF function in a lateral direction centered in the pixel with the maximum amplitude. The PSF in the axial direction is obtained by the same procedure. A different method is used to record the transverse resolution. The distribution of maximum echo pixels in ROI during vertical movement of the reflection ball is recorded from each frame.

To calculate the Lateral Resolution (LR) we analyse the PSF in the lateral direction. As LR we take the width of the amplitude peak in one half of the amplitude and recalibrate for the actual amplitude level.

Values $\mathrm{A}_{+\mathrm{LR}}\left(\mathrm{x}_{+}\right)$and $\mathrm{A}_{-\mathrm{LR}}\left(\mathrm{x}_{-}\right)$are found for the folowing conditions:

$$
\begin{aligned}
& \mathrm{x}_{+}>0 \text { and } \mathrm{x}_{-}<0 \\
& \qquad A_{ \pm L R}=\frac{A_{M A X}+A_{M I N}}{2} \quad[1 / 255]
\end{aligned}
$$

We can then express the LR corrected for difference between measured maximal amplitude $\mathrm{A}_{\mathrm{MAX}}$ and maximal possible amplitude 255 digitalisation units

$$
L R=\left(x_{+}-x_{-}\right) * \frac{255}{A_{M A X}} \quad[\mathrm{~mm}]
$$

$\mathrm{A}_{\mathrm{MAX}}$ is a peak amplitude in PSF

$\mathrm{A}_{\mathrm{MIN}}$ is minimal signal amplitude level in PSF (back ground noise level). 
To date we have been able to plot the LR characteristic over the scanning plane. This can differentiate separate scanning lines and even multiple focal areas for dynamic focussing systems. Currently we are working on accurate side lobe estimation. Our measuring system can detect malfunctions in dynamic focussing, size of aperture, time gain compensation function and/or transducer element failure.

The method itself is not as easy or as fast to use as tissue mimicking phantoms or 3D signal to noise ratio evaluation, but it provides accurate and objective numeric parameters corresponding to the quality of image at any specified point over the whole scanning area. It is also a very powerful tool when used in in combination with the other methods mentioned above.

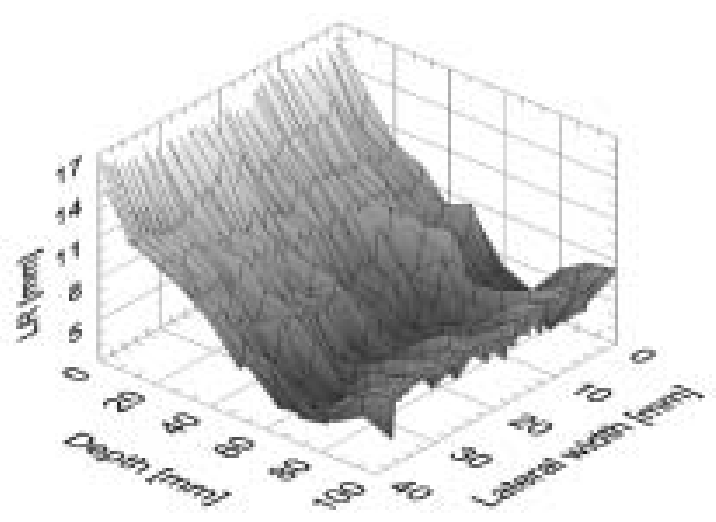

Fig. 1: LR characteristics of linear array transducer 3.5 $\mathrm{MHz}$, one focal piont used in depth $80 \mathrm{~mm}$.

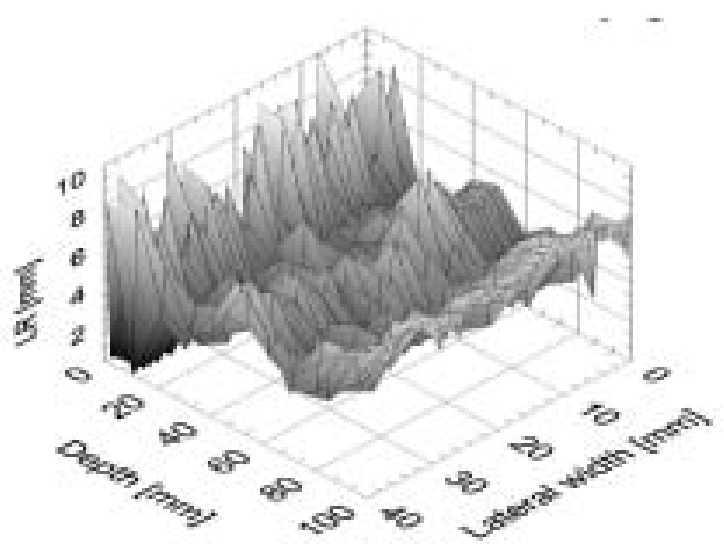

Fig. 2: LR characteristics of linear array transducer 3.5 $\mathrm{MHz}$, two focal pionts used in depth $30 \mathrm{~mm}$ and $80 \mathrm{~mm}$.

\section{Results}

We are able to display the shape of 2D LR distribution in the scan area of linear, convex and/or sector transducers in different working modes. The LR characteristics enable the measuring to detect analysis malfunction in dynamic focussing and size of aperture, time gain compensation function and its nonuniformity and/or transducer element(s) failure.

A sample of dynamic focussing effectivity comparison for use of one or two focal points is shown in Figs. 1 and 2. The tranducer used: linear array 3,5 $\mathrm{MHz}$, total imaging area of the transducer is $100 \mathrm{~mm}$ by $180 \mathrm{~mm}$, measured area $40 \mathrm{~mm}$ width and $100 \mathrm{~mm}$ depth.

\section{Conclusions}

A number of different sonographs using various transducers have been evaluated to date with promising results. Once the accuracy and reproducibility of this system are confirmed, it will be combined together with existing methods to create a method for measuring parameters of sonograph image quality and generate a databank of qualitative parameters of sonographs of various types and from different manufacturers.

The measuring system is protected by patent application PV 2003-3425.

Our work is supported by grant KONTAKT No.: 1P2004 ME720.

\section{References}

1. Alasaarela, E., Koivukangas, J.: Evaluation of image quality of ultrasound scanners in medical diagnostics. J Ultrasound Med 1990;1(9):23-34

2. Available on Internet: < http://www.ramsoft.biz/products/ultraiq/index.htm >

3. Available on Internet: $<\mathrm{http}: / /$ www.4sonora.com/test/firstcall.asp $>$

4. Kollmann Ch, Bergmann H, Trabold T, Zotz R. Ein Testobjekt zur Qualitätssicherung von 3D-Ultraschalgeraten. Z Med Phys 2001;1(11):45-52.

5. Rownd JJ, Madsen EL. Phantoms and automated system for testing the resolution of ultrasound scanners. Ultrasound Med Biol 1997;23(2):245-60.

6. Rozman J, Orel D, Václavík V et al. Modelová studie vyzařování ultrazvukových sond. Výsledky řešení projektu Grantové agentury ČR č. 102/00/0936, VUT v Brně, 2002, ISBN 80-214-2289-0.

7. Satrapa J, Doblhoff G. Automated quality control of diagnostic ultrasound appliances. Ultraschall Med 2002;23(2):123-8.

Ing. Ladislav Doležal, CSc., Palacky University Olomouc, Faculty of Medicine, Institute of Medical Biophysics, Hněvotínská 3, 77515 Olomouc, Czech Republic. e-mail: ladol@tunw.upol.cz 